\title{
Perfect Glued Graphs at Complete Clones
}

\author{
Chariya Uiyyasathian (Corresponding author) \\ Faculty of Science, Chulalongkorn University \\ Phyathai Rd., Patumwan, Bangkok 10330, Thailand \\ Tel: 66-2-218-5159Ｅ-mail: Chariya.U@Chula.ac.th \\ Supaporn Saduakdee \\ Faculty of Science, Chulalongkorn University \\ Phyathai Rd., Patumwan, Bangkok 10330, Thailand \\ Tel: 66-84-848-8410Ｅ-mail: aa_oo_rr@hotmail.com
}

\begin{abstract}
A graph $G$ is called perfect if the chromatic number and the clique number have the same value for every of its induced subgraph. A glued graph results from combining two vertex-disjoint graphs by identifying connected isomorphic subgraphs of both graphs. Such subgraphs are referred to as the clones.
\end{abstract}

We study the perfection of glued graphs whose clones are complete graphs. Our result generalizes the simplicial elimination ordering which is a characterization of chordal graphs.

Keywords: Perfect graphs, Glued graphs

\section{Introduction}

Let $G_{1}$ and $G_{2}$ be any two graphs with disjoint vertex sets. Let $H_{1} \subseteq G_{1}$ and $H_{2} \subseteq G_{2}$ be connected graphs such that $H_{1} \cong H_{2}$ with an isomorphism $f$. The glued graph of $G_{1}$ and $G_{2}$ at $H_{1}$ and $H_{2}$ with respect to $f$, denoted by $\underset{G_{1} \cong G_{2}}{G_{1} H_{2}}$, is the graph that results from combining $G_{1}$ with $G_{2}$ by identifying $H_{1}$ and $H_{2}$ with respect to the isomorphism $f$ between $H_{1}$ and $H_{2}$. Let $H$ be the copy of $H_{1}$ and $H_{2}$ in the glued graph. We refer to $H, H_{1}$ and $H_{2}$ as the clones of the glued graph, $G_{1}$ and $G_{2}$, respectively, and refer to $G_{1}$ and $G_{2}$ as the original graphs. The glued graph of $G_{1}$ and $G_{2}$ at the clone $H$, written $G_{1} \underset{H}{\Delta}$, means that there exist subgraph $H_{1}$ of $G_{1}$ and subgraph $H_{2}$ of $G_{2}$ and isomorphism $f$ between $H_{1}$ and $H_{2}$ such that $\underset{H}{G_{1} \triangleleft G_{2}}=\underset{H_{1} \cong f H_{2}}{G_{1}} G_{2}$ and $H$ is the copy of $H_{1}$ and $H_{2}$ in the resulting graph. We use $G_{1} \triangleleft G_{2}$ to denote an arbitrary graph resulting from gluing graphs $G_{1}$ and $G_{2}$ at any isomorphic subgraph $H_{1} \cong H_{2}$ with respect to any of their isomorphism.

A $k$-coloring of a graph $G$ is a coloring $f: V(G) \rightarrow S$, where $|S|=k$. A $k$-coloring is proper if adjacent vertices have different colors. The chromatic number of graph $G$, written $\chi(G)$, is the minimum number $k$ such that $G$ has a proper $k$-coloring. A clique of a graph $G$ is a complete subgraph of $G$. The clique number of a graph $G$, written $\omega(G)$, is the order of the largest clique of $G$. For any graph $G$, it is always true that $\chi(G) \geq \omega(G)$. A graph $G$ is called perfect if $\chi(F)=\omega(F)$ for every induced subgraph $F$ of $G$, and a graph is called imperfect if it is not perfect. An odd hole of $G$ is an induced subgraph of $G$ which is an odd cycle of length at least 5 . An odd antihole of $G$ is an induced subgraph of $G$ whose complement is an odd hole in $\bar{G}$. A graph having no odd hole and no odd antihole is called a Berge graph.

In 1972, Lovász proved the Perfect Graph Theorem, asserts that a graph is perfect if and only if its complement is perfect (Lovász , 1972). In 1961, Berge conjectured that a graph is perfect if and only if it is a Berge graph (Berge, 1961), affirmation well-known under the name of the Strong Perfect Graph Conjecture, and has just become a theorem since 2006 by Chudnovsky et al. (Chudnovsky et al., 2006). However, the proof was very 
long (179 pages), recently, Chudnovsky and Seymour replaced the final 55 pages with a new much shorter proof (Chudnovsky \& Seymour, 2009). This new theorem helps us to verify the perfection of our glued graphs.

Note that for vertex-disjoint graphs $G_{1}$ and $G_{2}, G_{1}+G_{2}$ stands for the disjoint union of $G_{1}$ and $G_{2}$. The join of $G_{1}$ and $G_{2}$, written $G_{1} \vee G_{2}$, is the graph obtained from the disjoint union $G_{1}+G_{2}$ by adding the edge set $\left\{u v: u \in V\left(G_{1}\right), v \in V\left(G_{2}\right)\right\}$.

It is possible that a glued graph of imperfect graphs is perfect and it is also possible that a glued graph of perfect graphs is imperfect, see Examples A and B.

Example A Perfect glued graphs of imperfect graphs:

Let $G_{1}=\overline{C_{2 n+1}}, G_{2}=K_{1} \vee C_{2 n-3}$ where $n \geq 4$ and $H=K_{1,2 n-3}$. Both $G_{1}$ and $G_{2}$ are not Berge graphs, hence they are imperfect by the Strong Perfect Graph Theorem. Observe that $\overline{G_{1} \triangleleft G_{2}} \cong P_{6}+\overline{K_{2 n-5}}$ which is perfect. By the Perfect Graph Theorem, we have that $G_{1} \underset{H}{\triangleleft} G_{2}$ is perfect. If $n=4$, then $G_{1}$ and $G_{2}$ are illustrated in Figure .

Note from Figure 1 that it is possible that a glued graph of simple graphs has multiple edges. However, multiple edges of a graph do not affect its the chromatic number and the clique number. Hence, we allow our glued graphs to have multiple edges.

Example B Imperfect glued graphs of perfect graphs:

Let $G_{1}=C_{2 n}, G_{2}=K_{1} \vee P_{2 n-2}$ where $n \geq 3$ and $H=P_{2 n-2}$. Both $G_{1}$ and $G_{2}$ are Berge graphs. Observe that $G_{1} \triangleleft G_{2}$ contains $C_{5}$, so it is not a Berge graph. By the Strong Perfect Graph Theorem, $G_{1}$ and $G_{2}$ are perfect but ${ }_{H}{ }_{H} \triangleleft G_{2}$ is imperfect. If $n=3$, then $G_{1}$ and $G_{2}$ are illustrated in Figure .

In this paper, we study the perfection of glued graphs of perfect graphs. Example B shows that a glued graph of perfect graphs may not be perfect. A condition is required to guarantee the perfection of a glued graph when the original graphs are perfect. Our main results reveal that the clone of the glued graph must be a complete graph in order to get the desired result.

Throughout the paper, $G_{1}$ and $G_{2}$ are graphs with disjoint vertex sets and the clone $H$ is a connected graph. We use symbol $G\left(u_{1}, u_{2}, \ldots, u_{n}\right)$ for a graph $G$ on the vertex set $\left\{u_{1}, u_{2}, \ldots, u_{n}\right\}$, and $\bar{G}\left(u_{1}, u_{2}, \ldots, u_{n}\right)$ for the complement of $G\left(u_{1}, u_{2}, \ldots, u_{n}\right)$. We use symbol $K_{n}\left(u_{1}, u_{2}, \ldots, u_{n}\right)$ for a complete graph on the vertex set $\left\{u_{1}, u_{2}, \ldots, u_{n}\right\}$, and $P_{n}\left(u_{1}, u_{2}, \ldots, u_{n}\right)$ and $C_{n}\left(u_{1}, u_{2}, \ldots, u_{n}\right)$ for a path and a cycle on the vertex set $\left\{u_{1}, u_{2}, \ldots, u_{n}\right\}$ and the edge set $\left\{u_{1} u_{2}, u_{2} u_{3}, \ldots, u_{n-1} u_{n}\right\}$ and $\left\{u_{1} u_{2}, u_{2} u_{3}, \ldots, u_{n-1} u_{n}, u_{n} u_{1}\right\}$, respectively. Other standard notations we follow West (West, 2001).

\section{Main Results}

When the clone $H$ is an induced subgraph of both $G_{1}$ and $G_{2}$, it follows that $G_{1}$ and $G_{2}$ are induced subgraphs of the glued graph $\underset{H}{G_{1} \triangleleft G_{2}}$. If $\underset{H}{G_{1} \triangleleft G_{2}}$ is perfect, then both $G_{1}$ and $G_{2}$ must be perfect. This can be concluded here:

Proposition 2.1 Let $G_{1}$ and $G_{2}$ be graphs containing $H$ as an induced subgraph. If $\underset{H}{G_{1} \triangleleft G_{2}}$ is a perfect graph, then both $G_{1}$ and $G_{2}$ are perfect.

The inverse of Proposition 2.1 is not true. Namely, if $H$ is not a complete graph, one can find perfect graphs $G_{1}$ and $G_{2}$ containing $H$ as an induced subgraph while $\underset{H}{G_{1} \triangleleft G_{2}}$ is not perfect.

Theorem 2.2 Let $H$ be a connected incomplete graph. If $H$ is a perfect graph, then there exist perfect graphs $G_{1}$ and $G_{2}$ containing $H$ as an induced subgraph such that $G_{1} \triangleleft G_{2}$ is not perfect.

Proof. Assume that $H$ is a perfect graph. Let $|V(H)|=r$. Let $H_{1}\left(u_{1}, u_{2}, \ldots, u_{r}\right)$ and $H_{2}\left(v_{1}, v_{2}, \ldots, v_{r}\right)$ be the copies of $H$ with an isomorphism $f: V\left(H_{1}\right) \rightarrow V\left(H_{2}\right)$ which is defined by $f\left(u_{i}\right)=v_{i}$ for all $i \in\{1,2, \ldots, r\}$. Let $P_{l}\left(u_{1}, u_{2}, \ldots, u_{l}\right)$ and $P_{l}\left(v_{1}, v_{2}, \ldots, v_{l}\right)$ be the longest induced paths of $H_{1}$ and $H_{2}$, respectively. Since $H_{1}$ and $H_{2}$ are not complete graphs, $l \geq 3$. Choose $G_{1}=H_{1} \vee K_{1}(z)$; a join graph between $H_{1}$ and a new vertex $z$, and choose $G_{2}=\left(H_{2} \vee K_{2}(x, y)\right)-\left\{x v_{l}, y v_{1}\right\}$. Then $G_{1}$ and $G_{2}$ are perfect. Consider $\underset{H_{1} \cong H_{2} H_{2}}{G_{1}}$, we see that the corresponding vertices of $v_{1}, x, y, v_{l}, z$ in $\underset{H_{1} \cong H_{f} H_{2}}{G_{1} \triangleleft G_{2}}$ form $C_{5}$. By the Strong Perfect Graph Theorem, $\underset{H_{1} \cong f H_{2}}{G_{1} \triangleleft G_{2}}$ is not perfect. 
The clone of a glued graph is called a complete clone if it is a complete graph.

The graph gluing at a complete clone preserves the perfection. Theorem 2.8 illustrates this fact and it is yielded by Lemmas 2.4 and 2.5 .

Remark 2.3 For graphs $G_{1}$ and $G_{2}$, we have

1. $\chi\left(G_{1} \triangleleft G_{2}\right) \geq \max \left\{\chi\left(G_{1}\right), \chi\left(G_{2}\right)\right\}$ and

2. $\omega\left(G_{1} \triangleleft G_{2}\right) \geq \max \left\{\omega\left(G_{1}\right), \omega\left(G_{2}\right)\right\}$.

In general, $\chi\left(G_{1} \triangleleft G_{2}\right) \leq \chi\left(G_{1}\right) \chi\left(G_{2}\right)$ (Promsakon \& Uiyyasathian, 2006). When the clone is a complete graph, the chromatic numbers of glued graphs do not exceed the chromatic numbers of their original graphs, see Lemma 2.4.

For a positive integer $r$, a glued graph at a complete clone, $\underset{K_{r}}{G_{1} \triangleleft G_{2}}$, denotes an arbitrary glued graph between graphs $G_{1}$ and $G_{2}$ at any clone which is isomorphic to $K_{r}$. For convenience, $K_{r}$ in our proofs always means the clone of the glued graph $G_{K_{r}}^{G_{1}} G_{2}$, not arbitrary subgraph $K_{r}$ in the glued graph .

Lemma 2.4 For graphs $G_{1}$ and $G_{2}, \chi\left(\underset{K_{r}}{G_{1} \bowtie G_{2}}\right)=\max \left\{\chi\left(G_{1}\right), \chi\left(G_{2}\right)\right\}$.

Proof. Let $\chi\left(G_{1}\right)=m$ and $\chi\left(G_{2}\right)=n$. Assume $m \geq n$. By Remark 2.3(1), it suffices to show that $\chi\left(G_{1} G_{K_{r}} G_{2}\right) \leq$ $m$. Let $a_{1}, a_{2}, \ldots, a_{m}$ be colors labeling vertices of $G_{1}$ by $f$ and $b_{1}, b_{2}, \ldots, b_{n}$ colors labeling vertices of $G_{2}$ by $g$. Note that any pair of vertices in $K_{r}$ must have different colors. Without loss of generality, for $i \in\{1,2, \ldots, r\}$, let $a_{i}$ and $b_{i}$ be colors of the corresponding vertices of $K_{r}$ in $G_{1}$ and $G_{2}$, respectively. Let $h: V\left({ }_{K_{r}} G_{1} G_{2}\right) \rightarrow$ $\left\{a_{1}, a_{2}, \ldots, a_{m}\right\}$ defined by

$$
h(v)= \begin{cases}f(v) & , \text { if } v \in V\left(G_{1}\right) \\ a_{i} & , \text { if } v \in V\left(G_{2}\right)-V\left(K_{r}\right) \text { and } g(v)=b_{i}\end{cases}
$$

Since the clone is a complete graph, $h$ is well-defined. To show that $h$ is proper, let $u$ and $v$ be vertices in $G_{1} \triangleleft G_{2}$ such that $u$ and $v$ are adjacent. If $u, v \in V\left(G_{1}\right)$, then $h(u)=f(u) \neq f(v)=h(v)$. If $u, v \in V\left(G_{2}\right)$, then $g(u) \stackrel{K_{r}}{=} b_{i}$ and $g(v)=b_{j}$ for some $i \neq j$, so $h(u)=a_{i} \neq a_{j}=h(v)$. Besides, vertices in $V\left(G_{1}\right)-V\left(K_{r}\right)$ and $V\left(G_{2}\right)-V\left(K_{r}\right)$ are not adjacent. Hence, $h$ is proper. That is, $\underset{K_{r}}{G_{1} \triangleleft G_{2}}$ has a proper $m$-coloring. Therefore, $\chi\left({ }_{K_{r}}^{G_{1} \triangleleft G_{2}}\right) \leq m$.

Unlike the chromatic number, we have not had an upper bound of the clique numbers of glued graphs in terms of the clique numbers of their original graphs. Promsakon conjectured that $\omega\left(G_{1} \triangleleft G_{2}\right) \leq \omega\left(G_{1}\right) \omega\left(G_{2}\right)$ (Promsakon, 2006).

In general, the graph gluing can join two non-adjacent vertices in the clone of an original graph, Consider graphs $G_{1}, G_{2}$ and $G_{1} \stackrel{\leftrightarrow}{H} G_{2}$ whose clone $H$ is shown as bold edges in Figure. We see that $b$ and $d$ are non-adjacent vertices in $G_{1}$ but the corresponding vertices of $b$ and $d$ in the glued graph are adjacent.

Since any pair of vertices in the complete clone are adjacent, there is no new edge created from the graph gluing. Thus all cliques in a glued graph at a complete clone are cliques in original graphs, so $\omega\left(G_{1} \triangleleft G_{2}\right) \leq \omega\left(G_{i}\right)$ for $i=1,2$. The following lemma is concluded.

Lemma 2.5 For graphs $G_{1}$ and $G_{2}, \omega\left(\underset{K_{r}}{G_{1} \triangleleft G_{2}}\right)=\max \left\{\omega\left(G_{1}\right), \omega\left(G_{2}\right)\right\}$.

The condition in Lemmas 2.4 and 2.5 that the clone must be a complete graph is necessary. Theorem 2.7 confirms.

Theorem 2.6 (Brooks, 1941) If $G$ is a connected graph other than a complete graph or an odd cycle, then $\chi(G) \leq \Delta(G)$ where $\Delta(G)$ denotes the maximum degree of $G$.

Theorem 2.7 Let $H$ be a connected graph. If $H$ is not a complete graph, then there exist $G_{1}$ and $G_{2}$ such that $\chi\left(\underset{H}{G_{1} \triangleleft G_{2}}\right)>\max \left\{\chi\left(G_{1}\right), \chi\left(G_{2}\right)\right\}$ and $\omega\left(G_{1} G_{H}\right)>\max \left\{\omega\left(G_{1}\right), \omega\left(G_{2}\right)\right\}$.

Proof. Assume that $H$ is not a complete graph. Let $|V(H)|=r$, so $r \geq 3$. Choose $G_{1} \cong K_{r}$ and choose $G_{2} \cong H \vee K_{1}$. Then $\underset{H}{G_{1} \triangleleft G_{2} \cong K_{r+1}}$. If $H$ is an odd cycle of length at least $5, \chi(H)=3<r-1$. Otherwise, $H$ 
is not an odd cycle of length at least 5, by Theorem 2.6, $\chi(H) \leq \Delta(H) \leq r-1$. Now, we have $\chi(H) \leq r-1$, so $\chi\left(G_{2}\right)=\chi\left(H \vee K_{1}\right) \leq(r-1)+1=r$. Hence, $\chi\left({ }_{1}^{G_{1} \triangleleft G_{2}}\right)=r+1>r=\max \left\{\chi\left(G_{1}\right), \chi\left(G_{2}\right)\right\}$. Since $H$ is not a complete graph and $|V(H)|=r$, we get $\omega(H) \leq r-1$. So, $\omega\left(G_{2}\right)=\omega\left(H \vee K_{1}\right) \leq(r-1)+1=r$. Therefore, $\omega\left(\underset{H}{G_{1} \triangleleft G_{2}}\right)=r+1>r=\max \left\{\omega\left(G_{1}\right), \omega\left(G_{2}\right)\right\}$.

For graphs $G_{1}$ and $G_{2}, G_{1} \cap G_{2}$ denotes the graph on the vertex set $V\left(G_{1}\right) \cap V\left(G_{2}\right)$ and the edge set $E\left(G_{1}\right) \cap E\left(G_{2}\right)$.

Theorem 2.8 For graphs $G_{1}$ and $G_{2}$,

$G_{1} \bowtie G_{2}$ is a perfect graph if and only if both $G_{1}$ and $G_{2}$ are perfect.

Furthermore, $\chi\left(\underset{K_{r}}{G_{1} G_{2}}\right)=\omega\left(\underset{K_{r}}{G_{1} \bowtie G_{2}}\right)=\max \left\{\omega\left(G_{1}\right), \omega\left(G_{2}\right)\right\}$.

Proof. Necessity follows from Proposition 2.1. For sufficiency, assume that $G_{1}$ and $G_{2}$ are perfect graphs. We will show that $\chi(F)=\omega(F)$ for every induced subgraph $F$ of $G_{K_{r}} G_{2}$. Let $F$ be an induced subgraph of $G_{1} \bowtie G_{r}$. If $F$ is disconnected, we consider the perfection of each component of $F$. We may assume that $F$ is connected. If $F$ has no vertex in $K_{r}$, then $F$ is an induced subgraph of either $G_{1}$ or $G_{2}$, so $\chi(F)=\omega(F)$. Assume that $F$ has at least one vertex in $K_{r}$. Let $F_{1}=F \cap G_{1}$ and $F_{2}=F \cap G_{2}$. Since $F_{1}$ is an induced subgraph of a perfect graph $G_{1}$, we get $\chi\left(F_{1}\right)=\omega\left(F_{1}\right)$. Similarly, $\chi\left(F_{2}\right)=\omega\left(F_{2}\right)$. Now, let $F_{r}=F \cap K_{r}$. Then $F_{r}$ is a complete graph. We have that $F=F_{F_{r}}$. By Lemmas 2.4 and $2.5, \chi(F)=\max \left\{\chi\left(F_{1}\right), \chi\left(F_{2}\right)\right\}$ and $\omega(F)=\max \left\{\omega\left(F_{1}\right), \omega\left(F_{2}\right)\right\}$, respectively. Hence, $\chi(F)=\omega(F)$. Therefore, $\underset{K_{r}}{G_{1} \triangleleft G_{2}}$ is perfect. Furthermore, $\chi\left(\underset{K_{r}}{G_{1} \triangleleft G_{2}}\right)=\omega\left(\underset{K_{r}}{G_{1} \triangleleft G_{2}}\right)=\max \left\{\omega\left(G_{1}\right), \omega\left(G_{2}\right)\right\}$.

If the clone is not a complete graph, it fails to be concluded the perfection of glued graphs of perfect graphs. It is illustrated by Theorems 2.9 and 2.10 .

Theorem 2.9 Let $H$ be a connected graph. If $H$ is not a complete graph, then there exist a perfect graph $G_{1}$ and an imperfect graph $G_{2}$ such that $G_{1} \underset{H}{\triangleleft} G_{2}$ is perfect.

Proof. Assume that $H$ is not a complete graph. Let $|V(H)|=r$, so $r \geq 3$. Let $H_{1}\left(u_{1}, u_{2}, \ldots, u_{r}\right)$ and $H_{2}\left(v_{1}, v_{2}, \ldots, v_{r}\right)$ be the copies of $H$ with an isomorphism $f: V\left(H_{1}\right) \rightarrow V\left(H_{2}\right)$ which is defined by $f\left(u_{i}\right)=v_{i}$ for all $i \in$ $\{1,2, \ldots, r\}$. Since $H_{2}$ is not a complete graph, there are at least 2 non-adjacent vertices, say $v_{1}$ and $v_{r}$. Choose $G_{1}=K_{r}\left(u_{1}, u_{2}, \ldots, u_{r}\right)$ and choose $G_{2}=\overline{C_{2 r-1}}\left(v_{1}, x_{1}, \ldots, v_{r-1}, x_{r-1}, v_{r}\right)$. Then $G_{1}$ is perfect but $G_{2}$ is not perfect. Since $H_{1}$ and $H_{2}$ are not complete graphs, $H_{1} \subseteq K_{r}\left(u_{1}, u_{2}, \ldots, u_{r}\right)$ and $H_{2} \subseteq K_{r}\left(v_{1}, v_{2}, \ldots, v_{r}\right)-v_{1} v_{r} \subseteq$

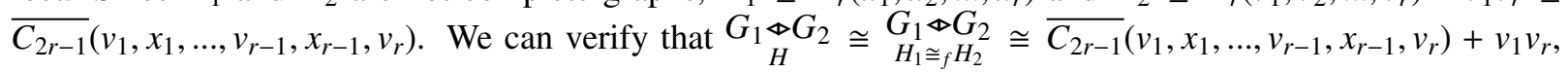
consequently, $\overline{G_{1} \bowtie G_{2}} \cong P_{2 r-1}$. Since $P_{2 r-1}$ is perfect, by the Perfect Graph Theorem, $\underset{H}{G_{1} \triangleleft G_{2}}$ is perfect.

Theorem 2.10 Let $H$ be a connected graph. If $H$ is not a complete graph, then there exist perfect graphs $G_{1}$ and $G_{2}$ such that $G_{1} \underset{H}{\leftrightarrow} G_{2}$ is not perfect.

Proof. Assume that $H$ is not a complete graph. Let $|V(H)|=r$, so $r \geq 3$. Let $H_{1}\left(u_{1}, u_{2}, \ldots, u_{r}\right)$ and $H_{2}\left(v_{1}, v_{2}, \ldots, v_{r}\right)$ be the copies of $H$ with an isomorphism $f: V\left(H_{1}\right) \rightarrow V\left(H_{2}\right)$ which is defined by $f\left(u_{i}\right)=v_{i}$ for all $i \in$ $\{1,2, \ldots, r\}$. Since $H_{2}$ is not a complete graph, there are at least 2 non-adjacent vertices, say $v_{1}$ and $v_{2}$. Choose $G_{1}=K_{r}\left(u_{1}, u_{2}, \ldots, u_{r}\right)$ and choose $G_{2}=\overline{C_{2 r+1}}\left(v_{1}, x_{1}, \ldots, v_{r}, x_{r}, x_{r+1}\right)-v_{1} v_{2}$. Then $G_{1}$ and $G_{2}$ are perfect. Since $H_{1}$ and $H_{2}$ are not complete graphs, $H_{1} \subseteq K_{r}\left(u_{1}, u_{2}, \ldots, u_{r}\right)$ and $H_{2} \subseteq K_{r}\left(v_{1}, v_{2}, \ldots, v_{r}\right)-v_{1} v_{2} \subseteq$

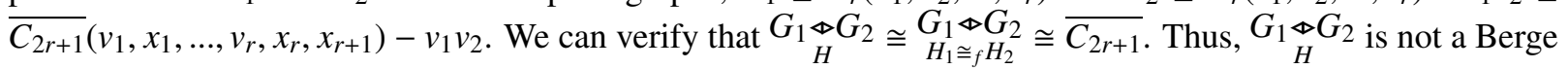
graph. By the Strong Perfect Graph Theorem, $\underset{H}{G_{1} \triangleleft G_{2}}$ is not perfect.

For a subset $S$ of $V(G)$, a neighborhood of $S$ in $G$, written $N_{G}(S)$ or $N(S)$, is the set of vertices in $V(G)-S$ which are adjacent to vertices in $S$. We use $G[S]$ and $G-S$ for the induced subgraph of $G$ on the vertex set $S$ and $V(G)-S$, respectively.

We now extend the simplicial (perfect) elimination ordering to a new definition as follows:

Definition 2.11 A subset $V_{i}$ of $V(G)$ is simplicial if its neighborhood in $G$ forms a clique. A simplicial set elimination ordering is an ordering $V_{1}, \ldots, V_{k}$ for deletion of nonempty vertex subsets so that each $V_{i}$ is a simplicial vertex subset of the remaining graph induced by $\bigcup_{t=i}^{k} V_{t}$ with $\left|V_{i}\right|=1$ or $\left|V_{i}\right|=2$ for all $i \in\{1,2, \ldots, k\}$, and $V_{1}, \ldots, V_{k}$ partitions $V(G)$. 
Note that a simplicial elimination ordering of a graph $G$ is a simplicial set elimination ordering of $G$ with $\left|V_{i}\right|=1$ for all $i \in\{1,2, \ldots, n(G)\}$.

Remark 2.12 For any nontrivial graph $G$, let $V_{1}, \ldots, V_{k}$ be a partition of $V(G)$. Let $G_{1}=G$, and for each $i \in\{2,3, \ldots, k\}$, let $G_{i}=G-\bigcup_{t=1}^{i-1} V_{t}$. If $V_{1}, \ldots, V_{k}$ is a simplicial set elimination ordering of $G$, then for each $i \in\{1,2, \ldots, k-1\}, G_{i}$ is a glued graph between $G_{i}\left[V_{i} \cup N\left(V_{i}\right)\right]$ and $G_{i+1}$ at a complete clone $G_{i}\left[N\left(V_{i}\right)\right]$.

Theorem 2.13 For a simple graph $G$, if $G$ has a simplicial set elimination ordering, then $G$ is a perfect graph.

Proof. Assume that $G$ has a simplicial set elimination ordering $V_{1}, \ldots, V_{k}$. Let $G_{1}=G$, and for each $i \in$ $\{2,3, \ldots, k\}$, let $G_{i}=G-\bigcup_{t=1}^{i-1} V_{t}$. Since $\left|V_{k}\right|=1$ or $2, G_{k} \cong K_{1}$ or $K_{2}$, so $G_{k}$ is perfect. By Remark 2.12, $G_{i}$ is a glued graph between $G_{i}\left[V_{i} \cup N\left(V_{i}\right)\right]$ and $G_{i+1}$ at a complete clone $G_{i}\left[N\left(V_{i}\right)\right]$, it is enough to claim that $G_{i}\left[V_{i} \cup N\left(V_{i}\right)\right]$ is perfect for all $i \in\{1,2, \ldots, k-1\}$. Let $i \in\{1,2, \ldots, k-1\}$. Let $C$ be an induced cycle in $G_{i}\left[V_{i} \cup N\left(V_{i}\right)\right]$. Since $N_{G_{i}}\left(V_{i}\right)$ forms a clique, at most 2 vertices in $N_{G_{i}}\left(V_{i}\right)$ can be in $C$. Together with vertices in $V_{i}, C$ has length at most 4 . Again, since $N_{G_{i}}\left(V_{i}\right)$ forms a clique, 2 vertices in $N_{G_{i}}\left(V_{i}\right)$ cannot be adjacent in the complement of $G_{i}\left[V_{i} \cup N\left(V_{i}\right)\right]$. Besides, each vertex in $N_{G_{i}}\left(V_{i}\right)$ must be adjacent to at least one vertex in $V_{i}$, so it can be adjacent to at most one vertex of $V_{i}$ in the complement of $G_{i}\left[V_{i} \cup N\left(V_{i}\right)\right]$. Thus, there is no cycle in the complement of $G_{i}\left[V_{i} \cup N\left(V_{i}\right)\right]$. Hence, $G_{i}\left[V_{i} \cup N\left(V_{i}\right)\right]$ contains no odd hole and no odd antihole. By the Strong Perfect Graph Theorem, we get $G_{i}\left[V_{i} \cup N\left(V_{i}\right)\right]$ is perfect. By Theorem 2.8, $G_{i}$ is perfect for all $i \in\{1,2, \ldots, k-1\}$.

The inverse of the theorem is not true, for instance, $C_{2 n}$, where $n \geq 3$, is perfect while it has no simplicial set elimination ordering. It is well-known that the simplicial elimination ordering characterizes a subclass of perfect graphs, namely the chordal graphs. It would be interesting if one could characterize a subclass of perfect graphs by the simplicial set elimination ordering.

\section{Acknowledgment}

The authors would like to thank the anonymous referee for helpful suggestions.

\section{References}

Berge C. (1961). Färbung von Graphen, deren sämtliche bzw. deren ungerade Kreise starr sind. Wiss. Z. Martin Luther Univ. Halle-Wittenberg Math.-Natur. Reihe, 10, 114-115.

Brooks R. L. (1941). On coloring the nodes of a network. Proc. Cambridge Phil.Soc., 37, 194-197.

Chudnovsky M., Robertson N., Seymour P. \& Thomas R. (2006). The strong perfect graph theorem. Ann. of Math., 164, 51-229.

Chudnovsky M. \& Seymour P. (2009). Even pairs in Berge graphs. J. Combin. Theory Ser. B, 99, 370-377.

Promsakon C. (2006). Colorability of Glued Graphs. Master Degree Thesis, Chulalongkorn University.

Promsakon C. \& Uiyyasathian C. (2006). Chromatic numbers of glued graphs. Thai J. Math., 4, No.3, 75-81.

Lovász L. (1972). A characterization of perfect graphs. J. Combin. Theory Ser. B, 13, 95-98.

West D.B. (2001). Introduction to Graph Theory. (2nd ed.). New Jersey: Prentice Hall. 

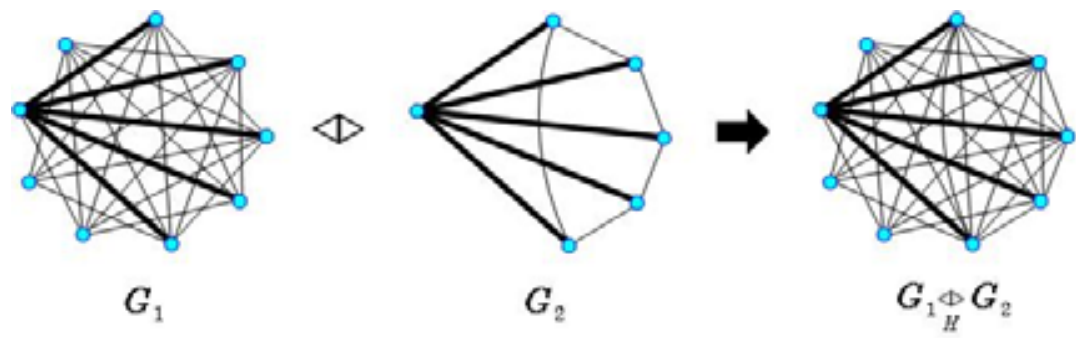

Figure 1. A perfect glued graph of imperfect graphs

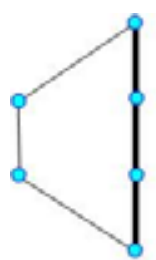

$G_{1}$

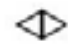

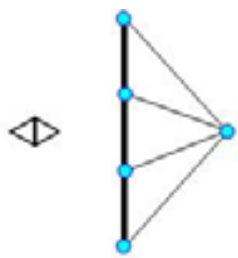

$G_{2}$

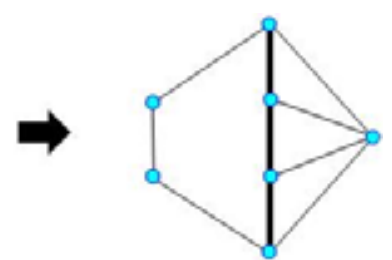

$G_{1}^{\oplus} G_{2}$

Figure 2. An imperfect glued graph of perfect graphs

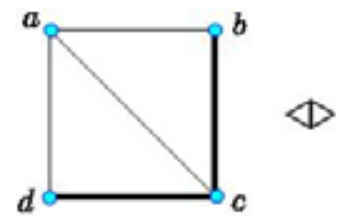

$G_{1}$

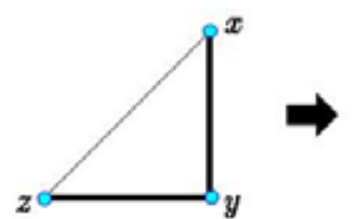

$G_{2}$

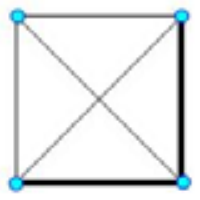

$G_{1} \Phi G_{2}$

Figure 3. A glued graph containing a new edge 